\section{Hypnagoger Fußtremor und alternierende \\ Beinmuskelbewegungen}

Helga Peter

Marburg, Deutschland

\section{Synonyme}

HFT und ALMA

\section{Englischer Begriff}

hypnagogic foot tremor (HFT) and alternating leg muscle activation (ALMA)

\section{Definition}

HFT äußert sich in meist einseitigen rhythmischen Bewegungen der Füße oder Zehen und tritt beim Schlaf-Wach-Übergang oder im leichten NREM-Schlaf (N1, N2) auf. Die Entäußerungen sind den Betroffenen nicht bewusst und werden als Zufallsbefund in der $\triangleright$ „Polysomnographie“ entdeckt; sie scheinen benigne und nicht behandlungsbedürftig zu sein. Häufig treten sie zusammen mit \ „Restless-Legs-Syndrom“ oder $\triangleright$,Schlafbezogene Atmungsstörungen“ auf.

ALMA zeigt sich in Form kurzer abwechselnder Aktivierungen des Musculus tibialis anterior beider Beine während aller Schlafstadien sowie vor oder nach einem $>$ „Arousal“". Die Bewegungen sind nicht immer sichtbar und sind ebenfalls ein polysomnographischer Zufallsbefund. Die Störung scheint benigne und nicht behandlungsbedürftig zu sein. Sie findet sich häufig zusammen mit periodischen Beinbewegungen (siehe $\triangleright$ „Periodische Beinbewegungen“) und Schlafbezogenen Atmungsstörungen; auch eine Assoziation mit der Einnahme von Antidepressiva wurde beschrieben. Die spezifischen polysomnographischen Kriterien für HFT und ALMA sind im • „AASM-Manual“ for the Scoring of Sleep an Associated Events definiert.

Die beiden Störungen werden derzeit in Einem genannt, da noch nicht geklärt ist, ob es sich um zwei voneinander unabhängige Entitäten handelt. Auch die Frage nach der klinischen Bedeutung von HFT und ALMA ist noch offen. Die Internationale Klassifikation der Schlafstörungen von 2014 ( „ICSD-3“) ordnet HFT und ALMA unter Isolierte Symptome und Normvarianten bei $\triangleright$ "Schlafbezogene Bewegungsstörungen“" ein. 